\section{Cuidado para el bienestar de personas con diabetes tipo 2 con heridas en miembros inferiores según la teoría de Kristen \\ Swanson}

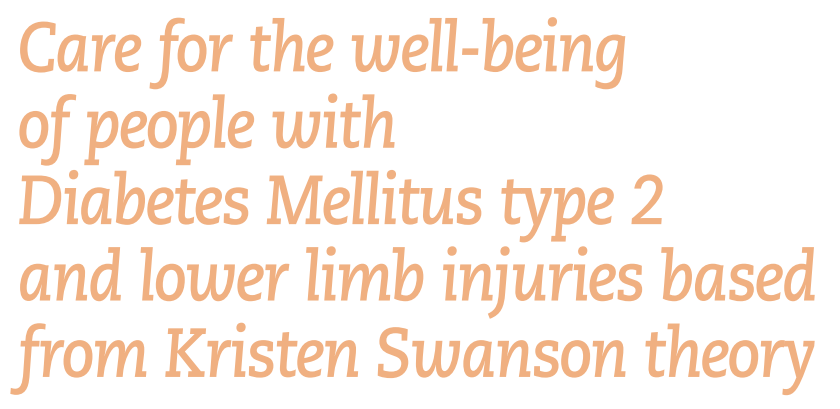

Ana María Murillo Salamanca ${ }^{1, *}$

Alejandra María Alvarado García'

1. Enfermera. Hospital San Rafael de Tunja. Magíster en Enfermería. Universidad de La Sabana. Chía. Cundinamarca. Colombia.

2. Enfermera. Doctora en Enfermería. Profesora Asociada. Universidad de La Sabana. Chía. Cundinamarca. Colombia.

*Autor para correspondencia.

Correo electrónico: anamursa@unisabana.edu.co (Ana María Murillo Salamanca).

Recibido el 22 de agosto de 2018; aceptado el 19 de julio de 2019.

\section{RESUMEN}

El cuidado brindado a las personas con diabetes mellitus tipo 2 (DM2) y heridas en miembros inferiores (MMII) se ha dirigido a la curación de las lesiones. Se requiere reorientar las acciones de los profesionales de enfermería para obtener una intervención más eficaz. Objetivo: Desarrollar un proyecto de gestión del cuidado, orientado por la teoría "Cuidado para el bienestar" de Kristen Swanson. Metodología: Se empleó la herramienta del marco lógico para la atención de los pacientes con DM2 que tienen heridas en MMII. Se presenta el proceso en los tres momentos de cuidado dispuestos por la teoría: conocer; mantener la fe y estar con; y hacer por y permitir. Resultados: Se evidencia el desconocimiento sobre la DM2 que tienen las personas y el trabajo realizado para superar esta limitación, acompañarlos en su desarrollo y favorecer su capacidad de cuidado, incluida la adherencia al tratamiento. Conclusiones: La intervención de "Cuidado para el bienestar" mostró resultados positivos en los indicadores propuestos dentro de los tres momentos y generó conductas que favorecieron la adherencia al cuidado de la salud y el de sus heridas, logrando así mejorar el bienestar de las personas con DM2 y un beneficio para la institución, al innovar en esquemas de cuidado dirigidos.

PALABRAS CLAVE: Diabetes mellitus, pie diabético, teoría de enfermería, promoción de la salud, educación en enfermería, teoría de Kristen Swanson.

\section{ABSTRACT}

Care that is given to those who have type II diabetes mellitus (DM2) and wounds in lower limbs mainly focuses on the healing of said wounds, but there is a call for nursing professionals to reorient their actions towards more efficient interventions. Aim: Developt a management project of care, focused on the theory of caring by Kristen Swanson. Methodology: This project, which uses the tool of a logic framework for the care of patients with lower limb wounds and DM2. The process is divided into three moments of care, which are given by the theory: to know; maintaining belief; and doing for and enabling the patient. Results: Lack of knowledge towards DM2 and towards the work put into overcoming this limitation, following the development of it, and encouraging the capacity of care, including adherence to treatment. Conclusions: The intervention of Care for wellness showed positive results in proposed indicators during the tree moments, which in turn generated conducts that favored the adherence to the care for their health and their wounds and, by doing so, achieving an improvement in the wellness of people with DM2 and a benefit for the institution, by innovating the schemes of directed care.

KEYWORDS: diabetes mellitus, Diabetic Foot, nursing Theory, health promotion, education, Kristen Swanson theory.

\section{У INTRODUCCIÓN}

A nivel mundial se ha visto un crecimiento acelerado de las enfermedades crónicas no trasmisibles relacionado con un cambio en los estilos y modos de vida de la población. Para el caso de la diabetes mellitus tipo 2
(DM2), se estima que en 2025 habrá cerca de 300 millones de personas en el mundo con esta enfermedad ${ }^{1}$.

La Organización Mundial de la Salud (OMS) determinó que la diabetes es responsable del $6 \%$ de las muertes a nivel mundial, y el $83 \%$ de esas muertes ocurren en países con ingresos medios y bajos; además, es 
una de las diez primeras causas de discapacidad en el mundo, puesto que aproximadamente 10 millones de personas con esta enfermedad sufren complicaciones incapacitantes, como enfermedades cerebrovasculares, ceguera, amputación de miembros inferiores y fallo renal ${ }^{2}$.

La DM2 es una de las cinco primeras causas de muerte y una de las diez primeras causas de consulta en adultos en Colombia. Con base en datos que se han recopilado en la última edición del IDF Diabetes Atlas de la International Diabetes Federation (IDF), se calcula que en el país hay aproximadamente 2 millones de personas con diabetes, de las cuales la gran mayoría es de tipo 2 .

Por consiguiente, esta enfermedad constituye un problema de salud pública que debe ser manejado en todos los niveles de atención y con estrategias de prevención en todas sus etapas. Además, estudios recientes sugieren que existe una memoria metabólica deletérea causada por la hiperglucemia, que obliga a controlar la diabetes de forma temprana y sostenida ${ }^{3}$.

Una de las complicaciones crónicas que con mayor frecuencia puede afectar a las personas con DM2 son las lesiones que se presentan en los miembros inferiores (MMII). Se denomina pie diabético al miembro inferior que presenta al menos una lesión con pérdida de continuidad de la piel (úlcera). El pie diabético, a su vez, se constituye en el principal factor de riesgo para la amputación de la extremidad ${ }^{4}$.

A pesar de ello, las lesiones en MMII suelen ser las más tardíamente diagnosticadas. Su prevalencia es difícil de establecer debido a la multiplicidad de métodos diagnósticos y a la heterogeneidad de las formas clínicas. Su evolución y gravedad se correlacionan con la duración de la enfermedad y el mal control metabólico. La detección depende de la sensibilidad de los métodos diagnósticos empleados ${ }^{5,6}$.

Los usuarios de las clínicas de heridas requieren seguimiento y control estrictos por parte de la agencia de enfermería, que involucren a su familia hasta la resolución completa de la herida y la reinserción de los pacientes a su cotidianidad; así mismo, es necesario un apoyo educativo que permita modificar estilos de vida para controlar los factores de riesgo presentes ${ }^{7}$.

Las personas con enfermedades crónicas atraviesan situaciones complejas ligadas a la rápida aparición de complicaciones, como es el caso de los pacientes con DM2 cuando no se tiene una adherencia a los tratamientos. Es por este motivo por el que se hace necesario el desarrollo de intervenciones donde se enfatice el autocuidado; se potencie un aprendizaje significativo; se facilité la escucha activa del paciente y se realicen entrenamientos para modificar la conducta, marcar objetivos realistas y alcanzables, con el apoyo de los materiales necesarios que mejor se adapten a las capacidades de las personas, y así, junto con el apoyo de los profesionales, se logre implementar prácticas de cuidado que favorezcan el bienestar de las personas ${ }^{8,9}$.

El cuidado de enfermería lleva implícita la identificación de problemas prioritarios en salud, la planeación, ejecución y evaluación de la atención de enfermería, determinados desde la perspectiva teórica donde se permita entender los fenómenos de cuidado. Las teorías de mediano rango (TMR) en la práctica de enfermería han ayudado a estructurar el conocimiento y los procesos de atención, ya que articulan de una manera sencilla conceptos abstractos para generar acciones puntuales que faciliten los procesos de cuidado; en este caso, llevar a cabo el bienestar de los pacientes. El bienestar ha sido interpretado como el proceso de vivir en un estado en el que los individuos se sienten integrados y comprometidos con el vivir y el morir. Por tal razón, al enfocar la salud como bienestar se debe tomar en cuenta el constante cambio, el crecimiento y la autorreflexión que experimentan las personas durante los procesos de salud-enfermedad ${ }^{10}$.

Durante la elección del marco conceptual se evaluaron diferentes teorías del bienestar con el fin de hallar la más congruente con las necesidades de las personas con heridas en MMII y se eligió la teoría de cuidado de Kristen Swanson, ya que denota el cuidado como "una forma de fomentar las relaciones con otro ser valioso hacia quien uno tiene un sentido personal de compromiso y responsabilidad" y permite el desarrollo de capacidades en la persona para ejercer acciones de autocuidado ${ }^{11}$.

La TMR de Kristen Swanson define que la enfermería es la disciplina conocedora de los cuidados para el bienestar de otros, siendo el cuidado una forma educativa de relacionarse con un ser apreciado hacia el que se siente un compromiso y una responsabilidad, haciendo que el paciente se sienta comprometido y logre asumir su responsabilidad en salud ${ }^{11}$. Los supuestos que se manejan en esta teoría se enuncian a continuación:

- Conocer: es esforzarse por entender los eventos en tanto tienen significado en la vida de las personas.

- Estar con significa estar emocionalmente presente con el otro. Incluye estar allí en persona, transmitir disponibilidad y compartir sentimientos sin abrumar a la persona cuidada.

- Hacer por significa hacer por otros lo que se haría por uno mismo, si fuera posible, incluyendo adelantarse a las necesidades, confortar, actuar con habilidad y competencia y proteger al que es cuidado, respetando su dignidad.

- Permitir pone énfasis en facilitar el paso del otro por las transiciones de la vida y los acontecimientos desconocidos, centrándose en el acontecimiento informando, explicando, apoyando, dando validez a sentimientos, generando alternativas, pensando las cosas detenidamente y dando retroalimentación.

- Mantener la fe se refiere a la capacidad del otro de superar un acontecimiento o transición y de enfrentarse al futuro con significado, creyendo en la capacidad del otro y teniéndolo en alta estima, manteniendo una actitud llena de esperanza, ofreciendo un optimismo realista, ayudando a encontrar el significado y estando al lado de la persona cuidada en cualquier situación ${ }^{12}$.

\section{METODOLOGÍA}

El desarrollo del proyecto de gestión empleó la metodología del marco lógico, la cual consiste en un esquema articulado de pasos que permiten tomar decisiones de manera rápida frente a problemas reales, dando soluciones en corto tiempo a problemas de la práctica ${ }^{15}$, basados en un plan detallado. Esta incluye el árbol de problemas, los objetivos, el referente teórico, la elaboración de la matriz del plan de acción, así como la evaluación y conclusiones.

Para este proyecto se planteó como problema la necesidad de generar una intervención para alcanzar el bienestar en el cuidado de las personas con DM2 que tienen heridas en MMII. El objetivo general es mejorar el cuidado dado a las personas con DM2 que tienen heridas en MM II en una unidad de consulta externa en Tunja Boyacá. Los objetivos específicos se realizaron a la luz de cada uno de sus componentes:

1. Conocer el significado que tiene para las personas con DM2 el evento de tener heridas en MM II en una unidad de consulta externa.

2. Desarrollar estrategias para mantener la fe y estar emocionalmente con las personas con DM2 para el manejo de sus heridas en MM II en una unidad de consulta externa.

3. Realizar intervenciones y fortalecer la capacidad de cuidado de las personas con DM2 y heridas en miembros.

Por ello, se tomó como base conceptual la teoría de cuidado para el bienestar de Kristen Swanson, con el fin de mejorar el cuidado dado a las personas con DM2 que tienen heridas en MMII. 
Tabla 1. Cronograma

\begin{tabular}{|c|c|c|c|c|c|c|c|}
\hline \multirow[t]{2}{*}{ Actividades } & \multicolumn{7}{|c|}{ Meses } \\
\hline & Febrero 2017 & Marzo 2017 & Abril 2017 & Mayo 2017 & Junio 2017 & Julio 2017 & Agosto 2017 \\
\hline \multicolumn{8}{|l|}{ Fase 1. Ajuste anteproyecto } \\
\hline \multicolumn{8}{|l|}{ Ajustes documento } \\
\hline \multicolumn{8}{|l|}{$\begin{array}{l}\text { Aprobación comité de ética } \\
\text { de las instituciones }\end{array}$} \\
\hline \multicolumn{8}{|c|}{ Fase $\mathbf{2}$ de captación de participantes } \\
\hline \multicolumn{8}{|l|}{ Desarrollo de la intervención } \\
\hline \multicolumn{8}{|c|}{ Fase 3. Análisis de la información } \\
\hline \multicolumn{8}{|l|}{ Revisión del documento } \\
\hline \multicolumn{8}{|l|}{ Entrega de documentos } \\
\hline Sustentación & & & & & & & \\
\hline
\end{tabular}

Criterios de inclusión: personas mayores de 50 años, con diagnóstico de DM2, que presentaran heridas en MMII asociadas a pie diabético, personas que no tuvieran antecedentes de enfermedad mental y aceptaran participar. La selección de los participantes se desarrolló con todas las personas que fueron atendidas en la clínica durante un período de 2 meses, y la intervención se ejecutó en 4 meses.

Criterios de exclusión: personas con heridas diferentes a pie diabético. Así mismo, se realizó con aval de las instituciones y tuvo en cuenta los lineamientos del Ministerio de Salud de Colombia y las pautas internacionales para la investigación biomédica, se utilizó el consentimiento informado, así como el respeto a la autonomía de los participantes ${ }^{13,14}$.

Para el desarrollo de la intervención se planteó el cronograma que se muestra en la tabla 1 .

\section{Plan de acción (tabla 2)}

Primer momento de cuidado: el primer momento de cuidado buscó conocer $^{16}$. Para desarrollar este proceso se utilizó la entrevista semiestructurada con el fin de identificar las necesidades de estos pacientes de la forma más cercana posible, para luego generar intervenciones que den respuesta a sus propios requerimientos ${ }^{17,18}$.

Segundo momento de cuidado: se desarrolló el proceso de mantener la fe, el cual hace referencia a "estar ahí", mostrando una disponibilidad continua y compartiendo sentimientos, ya sean de alegría o dolorosos a través del diálogo de saberes ${ }^{19}$. Esto permitió que el individuo se sintiera tranquilo, motivado al identificar la disponibilidad de la enfermera para brindarle cuidado. De igual manera, el permitir involucró el entrenamien-

Tabla 2. Plan de acción

\begin{tabular}{l|l}
\multicolumn{1}{|c|}{ Objetivos del proyecto } & \multicolumn{1}{c}{ Actividad } \\
\hline $\begin{array}{l}\text { 1. Momento de cuidado (Conocer) } \\
\text { Conocer el significado que tiene para }\end{array}$ & $\begin{array}{l}\text { Realizar entrevista exploratoria estructurada: } \\
\text { - Conocer lo que siente y percibe la persona a } \\
\text { las personas con diabetes mellitus tipo } \\
2 \text { (DM2) el evento de tener heridas en } \\
\text { miembros inferiores en una unidad de } \\
\text { consulta externa }\end{array}$ \\
\hline
\end{tabular}

\section{Momento de cuidado (Mantener la fe y estar con)}

Desarrollar estrategias para mantener la fe y estar emocionalmente con las personas con DM2 para el manejo de sus heridas en miembros inferiores en una unidad de consulta externa
Realización de un diálogo de saberes, a través de la actividad ¡Viviendo con heridas! para comprender cómo se cuidan las personas con DM2 Expresión de los saberes expresados por las personas con DM2 y heridas en miembros inferiores

\section{Subactividades}

Realizar un listado de preguntas sugeridas para la entrevista, referidas a partir de la búsqueda de literatura científica acerca de las necesidades de las personas con DM2

Solicitar autorización para realizar la entrevista, previa explicación a los participantes

Realización de la entrevista

Revisión de temas a través de la literatura científica para incluir en el diálogo de saberes:

- Diseño de material educativo, que permitiera apoyar el conocimiento de la población

- Realización de carteles con las preguntas a tratar en aras de facilitar la mayor comprensión de los asistentes

Diseño de material didáctico, en este caso modelos con lesiones en pie que permitieran mayor entendimiento y participación del adulto durante la sesión

Coordinación de área clínica para realizar la actividad

\section{Momento de cuidado (Hacer por y permitir)}

Realizar intervenciones de cuidado acordes con las necesidades de las personas con DM2 y heridas en miembros
Taller demostrativo

Permitir involucrar a la persona; entrenamiento, para permitir a las personas tener su experiencia de cuidado y vincular a la persona a generar nuevas alternativas 
to, información y explicación al otro, ayudarle y permitirle tener su propia experiencia y asistir al otro en el enfoque sobre asuntos importantes ${ }^{16}$. Dentro de la propuesta se desarrolló el diálogo de saberes, el cual, a través de un proceso comunicativo en el que se ponen en interacción dos lógicas diferentes (la del conocimiento científico y la del saber cotidiano), con una clara intención de comprenderse mutuamente, se logró el reconocimiento del otro como sujeto diferente, con conocimientos y posiciones diversas, logrando que los participantes expresaran sus saberes y diferentes cuidados ${ }^{20}$.

Tercer momento de cuidado: estar $\operatorname{con}^{18}$. Para desarrollar este concepto se realizaron intervenciones de enfermería acordes con las necesidades individuales, con el fin de ayudar al individuo a generar alternativas por medio de un taller demostrativo que permitió involucrar a la persona en un entrenamiento propio. Para ello se utilizaron estrategias basadas en el uso de modelos simulados en icopor en diferentes relieves, donde cada participante identificaba a través de ellos sus propias lesiones con un diseńo que le permitía recrear su herida. Vale la pena aclarar que se realizaron fotografías de las mismas con autorización previa de los participantes. Esto logró tener un contexto más real y así poder explicar a la persona las características de la herida; igualmente, el proceso y la técnica para realizar la curación de su herida en casa, dando opciones individualizadas, si en algún momento presentará inconvenientes con su herida. Luego, cada persona realizó una retroalimentación con su respectivo modelo sobre los cuidados aprendidos, y finalmente se realizó su curación.

Cada momento de cuidado tuvo una duración de 60 minutos, que empezó con un espacio de introducción sobre la actividad a realizar y al final un momento de retroalimentación con el objetivo de fortalecer la sesión educativa. En la última sesión se evaluó la actividad por medio de una encuesta de opinión sobre las actividades de cuidado realizadas.

\section{Resultados (tablas 3 y 4)}

Durante el proyecto fueron atendidas 5 mujeres y 4 hombres con DM2 y heridas en MMII, con un rango de edad entre 50 y 78 años, de los cuales
Tabla 3. Datos sociodemográficos. Formatos de cuidado para el bienestar de personas con diabetes mellitus tipo 2 y heridas en miembros inferiores

\begin{tabular}{|l|l|l|l|}
\hline Variables sociodemográficas & n & $(\%)$ \\
\hline Femenino & & 5 & 55 \\
\hline Masculino & Casado & 4 & 45 \\
\hline Estado civil & Viudo & 7 & 78 \\
\hline & Unión libre & 1 & 11 \\
\hline Procedencia & Urbana & 1 & 11 \\
\hline & Rural & 8 & 88 \\
\hline Escolaridad & Primaria & 1 & 12 \\
\hline Tiempo de aparición & Bachillerato & 8 & 88 \\
\hline de las heridas 2 años & 3 a 4 años & 1 & 12 \\
\hline & 5 o más años & 3 & 34 \\
\hline
\end{tabular}

Fuente: Elaboración propia

uno vive en la zona rural. El tiempo de aparición de las heridas en MMII está comprendida entre 2 y 5 años. De las personas participantes, tres en la actualidad estaban trabajando y los demás se dedicaban a realizar tareas cortas para colaborar en el hogar. Estas personas asistían como mínimo 2 veces por semana al servicio de clínica de heridas para la realización de su curación.

En el proceso de desarrollo de los momentos de cuidado, a cada persona se le informaba de los diferentes cuidados que debía emprender para mantener el control de la DM2 y evitar así la aparición de otras complicaciones. Asimismo, se identificaban pautas de cuidado para fa-

Tabla 4. Resultados a partir de indicadores

\section{Momento de cuidado \\ Indicador de estructura}

1. Conocer

\section{Mantener la fe y estar con}

\section{Hacer por y permitir} manejo de sus heridas dar educación
No se indagaba en los pacientes acerca de sus conocimientos sobre la enfermedad y de las necesidades de cuidado en aras de favorecer la adherencia a su tratamiento
Indicador de proceso

Las enfermeras, durante la consulta, realizan ahora una entrevista a los pacientes con el fin de conocer sus propias necesidades y dar respuesta a lo requerido por ellos

No se disponían espacios donde el grupo de pacientes pudieran socializar sus experiencias en torno a la enfermedad y al

No se disponía de material educativo para
Se tienen en cuenta espacios donde grupos de personas con DM2 comparten sus prácticas y se apoyan en el cuidado Las enfermeras educan a partir de lo referido por los participantes durante la socialización, con el objetivo de reforzar conductas y mejorar conocimientos

Se cuenta con un taller organizado, con material apropiado que consta del uso de diferentes modelos de heridas realizadas en icopor que simulan las propias heridas de los pacientes, con el fin de facilitar su comprensión

\section{Indicador de producto}

El nivel de participación era del $30 \%$ y al finalizar el proyecto fue de $85 \%$

El nivel de participación al inicio era de 20\% al finalizar de $100 \%$

La satisfacción con el cuidado se desconocía y ahora es de $98 \%$ 
vorecer el proceso de cicatrización de sus heridas y para que en caso de que la curación se dañara evitar la infección de la misma.

Después de llevar a cabo cada una de las estrategias, a la luz de la teoría de bienestar se logra identificar, durante el primer momento, cuándo se realizaron las entrevistas, y se hace el respectivo análisis, manteniendo el rigor y la privacidad de los datos. A continuación, se exponen algunos de los testimonios de los participantes en cuanto a la aparición de sus heridas:

"La herida fue puro descuido, no tenía conciencia de lo que tengo E5".

"Por no saber qué es la enfermedad y cómo cuidarse a tiempo antes de tener" E1.

"Las heridas que lo hacen ver a uno sucio..., la gente se aleja, porque eso suelta agua y piensan que uno es el sucio E3".

Esto podría ilustrar cómo las heridas se producen por desconocimiento de la enfermedad, siendo resultado de una baja información sobre la enfermedad. Así mismo, se evidencia cómo la herida no solo tiene una representación fisiológica en las personas con DM2, sino también un componente de orden psicosocial donde se ve afectada la autoimagen, la autoestima y el rol. El nivel de participación frente al conocimiento era del $30 \%$, y al finalizar fue del 85\%; los pacientes se sentían más seguros de expresar sus sentimientos.

En el segundo momento de cuidado se realizó un diálogo de saberes donde se dio apertura a los participantes para que lograran evidenciar el proceso de mantener la creencia y permitir. Los participantes pudieron expresar, desde su saber, cómo cuidaban sus heridas. Se orientó a las personas hacia la búsqueda de alternativas de todo tipo para sanar sus heridas, incluso sus tradiciones en salud dadas desde la cultura:

“... con yerbas como paico, yerba mora, caléndula E2".

"Me acuerdo que el agua oxigenada ardía mucho, pero me dejaba la herida blanca y pensé que así me sanaba E4".

"Creo que me bañe los pies con todo lo que me dijeron, es por el desespero de volver a ser el mismo E4".

Se evidencia en este ejemplo cómo el saber cultural tiene un papel importante, lo mismo que el compartir experiencias, ya que ellos transmiten lo que aprenden dentro de su contexto. Es así como entender el componente cultural y el detenerse a dialogar durante el momento de la intervención sobre las rutinas de cuidado tiene un papel importante con el fin de conocer las prácticas, para explicar la pertinencia o no de estas. El nivel de participación al inicio era de $20 \%$, se notaban muy tímidos durante las sesiones, pero al finalizar, este porcentaje mejoró al $100 \%$, ya que todos se sentían más dispuestos y receptivos (fig. 1).

El tercer momento de cuidado fue un espacio de aprendizaje y entrenamiento para comprender el cuidado específico para cada una de las heridas del paciente, con el ánimo de extender el cuidado en su domicilio y tener una opción en caso de que la curación se dañe o bien no se pueda asistir a la siguiente consulta de curación en el centro hospitalario, de modo que no se afecte el proceso de cicatrización de la herida.

Las personas tuvieron la experiencia de conocer su herida a partir de los modelos de MM II y la foto de su herida, realizaron su curación, y a partir de la experiencia expresaron la evolución de su herida y los cuidados que debían realizar. Esta actividad permitió que el paciente se sintiera más seguro sobre lo que estaba haciendo, al tener la retroalimentación y acompańamiento permanente por parte el profesional de enfermería, logrando así que las personas con DM2 reconocieran qué actividades contribuyen a su salud y recuperación, fomentando su bienestar y autonomía en el cuidado de sus heridas.

Se observa una mejora significativa en la experiencia de cuidado de las personas referente a los conocimientos sobre la DM2. Antes de la

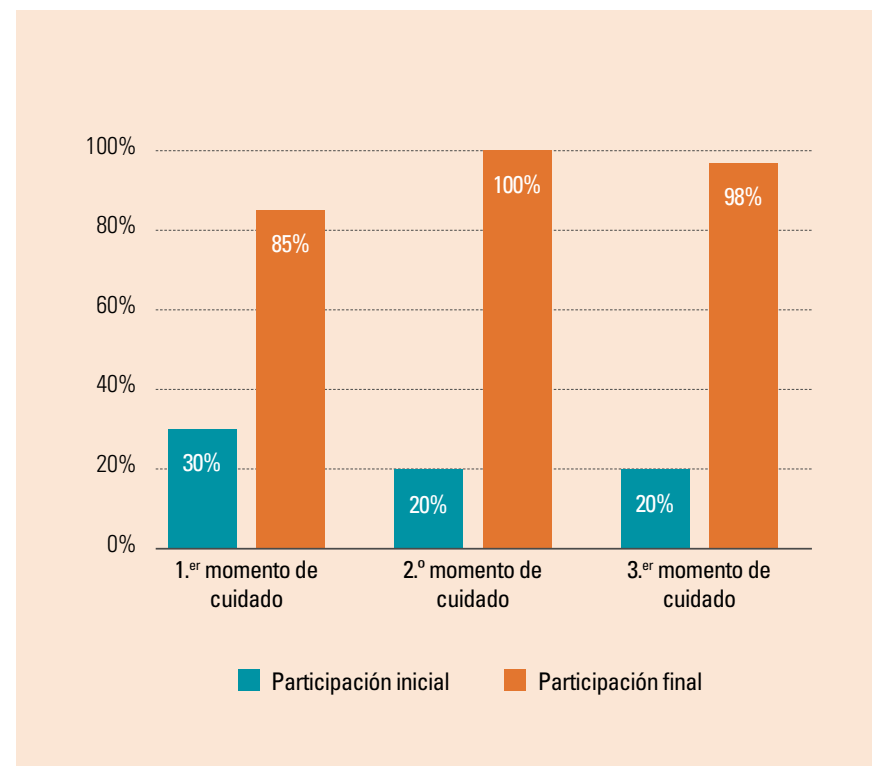

Figura 1. Nivel de participación en la intervención de cuidado para el bienestar.

intervención, las personas no sabían por qué se presentaron esas heridas en su cuerpo; desconocían cómo el descontrol metabólico influía en su aparición y cronicidad. Después de la intervención se evidencia cómo las personas relacionan los alimentos que deben consumir y los pasos para el autocontrol de la enfermedad, asisten a la cita de curación con su registro de glucómetro y se notan optimistas en el avance de sus heridas.

La satisfacción con el cuidado brindado al inicio de la intervención se desconocía. Una vez realizado el proyecto de gestión, esta fue del 98\%; las personas manifestaban la importancia de sentirse útiles en el proceso de sus curaciones; se les escuchó e involucró en su autocuidado $\mathrm{y}$ al mismo tiempo se tuvo en cuenta su vivencia para proponer nuevas formas de atención. Por otro lado, se organizó la forma de proporcionar el cuidado en la institución a partir de la teoría de Swanson en las tres etapas que se enunciaron anteriormente.

\section{У DISCUSIÓN}

Tras la realización de este estudio, y con el fin de desarrollar el presente apartado, se han podido apreciar diferentes investigaciones que nos permiten contrastar el valor de emprender acciones de cuidado a partir de analizar e identificar las necesidades individuales, para resolver dudas, miedos, debilidades y así potenciar el desarrollo de habilidades y generar intervenciones de enfermería más acordes.

Así, el modelo de Kristen Swanson, adaptado al cuidado de enfermería en una investigación dirigida a personas adultas mayores con enfermedad crónica, considera esencial la valoración integral y la interpretación de sus vivencias, ya que estas se refieren a la comprensión de lo que son, lo que ellas consideran sobre la atención y las estrategias de afrontamiento de sus conflictos diarios. El modelo de atención propuesto se basa en los sentimientos, las actitudes y el conocimiento de adultos mayores reveladas por la historia de su vida pasada, presente y perspectivas de futuro ${ }^{20}$.

Posada y Mora proponen brindar un cuidado humanizado a partir de "conocer" al "otro" desde lo humano, para que el cuidado propuesto tenga significado para la persona, lo que implica que la enfermera ha de tener capacidad para estar emocionalmente presente, hacer todo lo que la persona haría por sí misma si le fuera posible, pero sin afectar a su 
dignidad o sus creencias, posibilitando nuevamente su independencia, ya que las reacciones humanas de índole física, biológica, emocional y psicológica pueden ser producto de una situación que afecta al bienestar del ser humano y demandan cuidados centrados en la persona, es decir, soluciones acordes a la enfermedad, expectativas y esperanza que la persona tenga en ese momento ${ }^{21}$.

De este modo, analizar las vivencias y los conocimientos previos no solo permite una identificación de las necesidades, es una herramienta que permite definir un rol activo de las personas en el cuidado a partir de la generación de autopautas, y el rescate de los cuidados favorables inmersos en el compendio del cuidado tradicional. Según González y Verdú, es primordial conocer la percepción del efecto de la enfermedad, de la intervención o del tratamiento sobre la vida de las personas, para direccionar con certeza el abordaje más idóneo ${ }^{22}$. Igualmente, se requiere plantear intervenciones con metas medibles y evaluables, donde se ponen en juego verdades, saberes y sentimientos en la búsqueda de acuerdos que aportan a la construcción de un conocimiento colectivo y favorecen el cuidado de enfermería, el cual se ha definido como el encuentro entre seres humanos (educandos y educadores), en este caso concreto paciente-enfermera, donde los dos se construyen ${ }^{23,24}$.

Seguidamente se requiere evaluar lo aprendido a partir de valorar las destrezas adquiridas. Este espacio es crucial en el proceso de atención de la persona con heridas, ya que las intervenciones deben ser evaluadas y medibles para mirar los resultados del cuidado y es el instante para reafirmar y corregir lo aprendido. Por tanto, la enfermera debe trabajar en intervenciones específicas que permitan abordar a la persona y llevarla a la modificación de conductas de cuidado mediante prácticas educativas de salud que se relacionan con una mayor prevención de las complicaciones por medio de su participación activa en el cuidado, lo cual posibilita a la persona convivir mejor con su condición y esto genera bienestar ${ }^{25}$. En este proceso, la familia requiere de un acercamiento progresivo con los profesionales de la salud, para la construcción conjunta de conocimiento y toma de decisiones sobre el cuidado de la salud ${ }^{26}$.

Reafirmando lo anterior, Compeán, Gallegos y colaboradores describen cómo la implementación de intervenciones cognitivo-educativas multidisciplinarias dirigidas al adulto puede favorecer la adquisición individual de conocimiento y ser responsables por conductas saludables de autocuidado, lo que conduciría no solo a brindar intervenciones de enfermería congruentes, sino a generar el empoderamiento del cuidado de la enfermedad ${ }^{27}$. Algunos componentes de estas intervenciones se concentran en el asesoramiento al paciente; las estrategias de simplificación posológica o la potenciación de la comunicación entre el profesional de la salud y el paciente parecen ser herramientas eficaces en la mejora de la adherencia en este grupo poblacional ${ }^{8,9}$. Las intervenciones de enfermería se dirigen a cuidar a las personas de forma integral con el propósito de involucrarlas en el cuidado, otorgando a la persona la posibilidad de vivenciar una función primordial en el proceso de salud-enfermedad.

Para el planteamiento de intervenciones en enfermería se han de utilizar las TRM, ya que estas son aplicables a los problemas visibles en la práctica y permiten evidenciar fenómenos que usualmente no se tienen en cuenta durante el cuidado de enfermería a las personas con heridas. En este caso particular, al aplicar la teoría del bienestar se logra identificar y aplicar conceptos específicos "el conocer, el hacer por y el permitir" para brindar un cuidado integral y favorecer la relación enfermera paciente ${ }^{26}$.

El desarrollo disciplinar en enfermería requiere de la aplicación de TRM en la práctica como un herramienta útil y sencilla que responde a sus necesidades disciplinares, y sin duda se puede asegurar que provienen, en su mayoría, de la combinación de la práctica y la investigación dando forma a varias expresiones de esta como son intervenciones a partir de diagnósticos, elaboración de guías y protocolos clínicos, definición de estándares de cuidado y otros indicadores empíricos que expresan las características de la práctica ${ }^{28}$.

Es un reto para el profesional de enfermería trascender para brindar un cuidado, que cumpla con los requerimientos del paciente y de su familia, basado en los principios humanos que guían la profesión y permiten reciprocidad. El cuidado de enfermería conceptualizado como: "la auténtica presencia de las enfermeras con el otro, quien es reconocido como persona que vive y crece en el cuidado. La enfermera intenta conocer al otro como persona que cuida de sí mismo y busca comprender cómo puede ayudar, apoyar y dar fortaleza a la persona" ${ }^{29}$.

Por último, es importante recalcar que el planteamiento de intervenciones de enfermería acordes con las necesidades de las personas considera a las enfermeras como proveedoras de cuidado a estas personas (individuos o agregados) que enfrentan anormalidades en su salud; en ese hacer se reflejan los valores, historia, experticia, conocimiento, universalidad y pasión de la enfermería, con una integración de la ciencia, la persona, la preocupación por la humanidad y el cuidado ${ }^{30,31}$.

\section{$\searrow$ CONCLUSIONES}

La aplicación de la propuesta cuidado para el bienestar de personas con DM2 y heridas en MM II según la teoría de Kristen Swanson permitió crear intervenciones de enfermería más acordes con las necesidades de los pacientes, condujo a empoderar a las personas en su autocuidado y generó una nueva alternativa para encaminar la atención de las personas en la clínica de heridas.

La propuesta permitió explorar el campo social y humano de las personas, ya que a través del conocer (para aprender la realidad del otro, se visibilizan los sentimientos, el dolor y la esperanza de cada persona); estar con (escuchar al otro e identificar todo aquello que tiene para dar facilita los lazos de diálogo con la persona de cuidado); hacer para (brindando espacios fuera de la consulta de clínica de heridas, para fortalecer conocimientos sobre la herida; permitir que la persona de cuidado exprese sus dudas y reconozca en sus heridas la fortaleza para luchar por el control de su enfermedad; mantener la creencia, para valorar todos los cuidados que han efectuado en busca de bienestar, y en mantener la esperanza en las personas de que las acciones de cuidado, conducirán al mejoramiento de su herida.

Para la disciplina de enfermería este proyecto puso de manifiesto la importancia de forjar intervenciones más íntegras, que no solo partan del qué hacer, sino que se dirijan a innovar en estrategias que permitan mejorar el autocuidado de las personas.

La intervención "Cuidado para el bienestar" mostró resultados positivos en los indicadores propuestos dentro de los tres momentos, generando conductas que favorecieron la adherencia al cuidado de la salud y al de las heridas, logrando así mejorar el bienestar de las personas con DM2 y un beneficio para la institución, al innovar en esquemas de cuidado dirigidos hacia las necesidades sentidas de una población.

Las TRM son la herramienta adecuada para sustentar las intervenciones de cuidado de enfermería, ya que generan explicaciones sobre el porqué de los fenómenos íntimamente ligados al quehacer cotidiano de enfermería. Igualmente, las TRM orientan el reconocimiento y abordaje de los conceptos fundamentales del cuidado de los pacientes en la asistencia diaria. En pocas palabras, interpretar los hallazgos guía las intervenciones de enfermería con el conocimiento específico de la disciplina de Enfermería.

Los resultados de este estudio permiten probar que las intervenciones de enfermería sobre la persona pueden ser medidos e influir en 
resultados del cuidado, específicamente en el conocimiento que obtiene la persona sobre la forma de cuidarse, y al mismo tiempo permite a la disciplina de enfermería demostrar su papel central en el proceso educativo.

\section{Conflicto de intereses}

Los autores han declarado que no existen conflictos de interés personal, financieros, políticos ni académicos en relación con los resultados de esta investigación y su publicación.

\section{У BIBLIOGRAFÍA}

1. Rodrigues Helmo F, Dias FA, Bonato Zuffi F, Borges MF, Halla Jorge Lara $B$, Ferreira LA. Cuidado de los pies: conocimiento de los individuos con diabetes mellitus. Enferm. glob. [Internet]. 2014 [citado 5 de octubre de 2018);1335:41-51. Disponible en: http://scielo.isciii.es/scielo.php?script=sci_arttext\&pid=S1695-61412014000300003\&lng=es

2. OMS | Informe mundial sobre la diabetes. WHO [Internet]. 2016 [citado 11 de diciembre de 2017]. Disponible en: http://www. who.int/diabetes/global-report/es/

3. Campos de Aldana MS, Moya Plata D, Mendoza Matajira JD, Duran Niño EY. Las enfermedades crónicas no transmisibles y el uso de tecnologías de información y comunicación: revisión sistemática. Rev Cuid [Internet]. 2014 [citado 5 de octubre de 2018];5(1):661-9. Disponible en: http://www.scielo.org.co/scielo. php?script=sci_arttext\&pid=S2216-09732014000100010\&lng

4. Who. Global health risks: mortality and burden of disease attributable to selected major risks Geneva: WHO Press; 2016. Disponible en: http://www.who.int/diabetes/country-profiles/es/

5. Atlas de la Federación Internacional de diabetes DF news [Internet]. [citado 11 de diciembre de 2017]. Disponible en: https:// www.idf.org/news/94:new-idf-figures-show-continued-increase-in-diabetes-across-the-globe,-reiterating-the-need-for-urgent-action.html

6. Análisis de situación de salud ASIS. Colombia, 2016. Disponible en: https://www.minsalud.gov.co/sites/rid/Lists/BibliotecaDigital/RIDE/NS/ED/PSP/asis-colombia-2016.pdf

7. Organización Panamericana de la Salud. Guías ALAD de diagnóstico, control y tratamiento de diabetes mellitus tipo 2. Washington, DC: OPS; 2013. [Online]. Disponible en: http://www. paho.org/mex/index.php?option=com_docman\&task=doc_download\&gid=424\&itemid

8. Giraudo N, Chiarpenello J. Actualización: Educación para la salud basada en la comunidad (primera entrega). Community based health education. EVIDENCIA - Actualización en la Práctica ambulatoria [Internet] [citado 11 de diciembre de 2017];2011;14(4):1428. Disponible en: http://www.fundacionmf.org.ar/files/16 .pdf

9. Colchero Camacho IM. Intervenciones para la mejora de la adherencia al tratamiento en pacientes pluripatológicos. Enferm Clin [Internet]. 2016 [citado 11 de diciembre de 2017];26(5):3257. Disponible en: http://linkinghub.elsevier.com/retrieve/pii/ \$1130862116300328.

10. Guerrero Núñez S, Valenzuela Suazo S, Cid Henríquez P. Cuidado de enfermería en personas con diabetes mellitus tipo 2 según la Teoría de la Atención Burocrática. Aquichan [S.I.] [acceso 5 de octubre de 2018]. 2018;18(1):20-31. Disponible en: <http://aquichan.unisabana.edu.co/index.php/aquichan/article/view/6929 . Fecha de acceso: 05 oct. 2018
11. Rodríguez Campo VA, Valenzuela Suazo S. Teoría de los cuidados de Swanson y sus fundamentos, una teoría de mediano rango para la enfermería profesional en Chile. Enferm. glob [Internet] 2012;11(n28). Disponible en: http://scielo.isciii.es/scielo.php?pi$\mathrm{d}=$ S1695-61412012000400016\&script=sci_arttext

12. Méndez JFC, Garzón JXR. Aplicación de la Teoría de Kristen Swanson en el cuidado de pacientes con enfermedad coronaria: una experiencia desde la docencia Colombia: Editorial UNIMAR: 2016

13. República de Colombia. Ministerio de Salud. Resolución $n^{0}$ 008430 de 1993 (4 de octubre de 1993) Por la cual se establecen las normas científicas, técnicas y administrativas para la investigación en salud. Bogotá, Colombia. Disponible en: https://urosario.edu.co/Escuela-Medicina/Investigacion/Documentos-de-interes/Files/resolucion 008430_1993.pdf

14. Stepke F. Pautas éticas internacionales para la investigación biomédica en seres humanos. Ginebra: Council for International Organizations of Medical Sciences (CIOMS); 2002. Disponible en: http://www1.paho org/Spanish/BIO/CIOMS.pdf

15. Moreno Maria E, Muñoz De Rodriguez L. De la teoría de enfemería a la práctica: Experiencias con proyectos de gestión del cuidado. Facultad de Enfermería y Rehabilitación. Bogotá: Universidad de La Sabana; 2016. DOl: http://doi.org/10.5294/978958-1204-13-7

16. Kalfoss M, Owe J. Empirical Verification of Swanson's Caring Processes Found in Nursing Actions: Systematic Review Swanson's Middle Range Caring Theory. Open J Nurs [Internet]. 2015 [citado 11 de diciembre de 2017];5(5):976-86. Disponible en: http:// www.scirp.org/journal/ojn

17. Politi MC, Han PKJ, Col NF. Communicating the Uncertainty of Harms and Benefits of Medical Interventions. Med Decis Mak [Internet]. 2007 [citado 11 de diciembre de 2017];27(5):681-95. Disponible en: http://journals.sagepub.com/ doi/10.1177/0272989X07307270

18. LoBiondo-Wood G, Haber J. Nursing research: Methods and critical appraisal for evidence-based practice. St. Louis: Mosby Elsevier, 2013. $616 p$

19. Swanson KM. Nursing as Informed Caring for the Well-Being of Others J Nurs [Internet] 1993 [citado 11 de diciembre de 2017];25(4):352-7. Disponible en: http://nursing.unc.edu/files/2012/11/ccm3_032549.pdf

20. Rojas J, García M. Adaptación del modelo de Kristen Swanson para el cuidado de enfermería en adultas mayores. Texto contexto - enferm. 2018;27(4):e0660017. Disponible en: https://www.scielo. $\mathrm{br} / \mathrm{sciel}$.php?script=sci_arttext\&pid=S0104-07072018000400316

21. Posada M, Mora B. Interpretación de la teoría de Kristen Swanson para un cuidado humanizado. Metas Enferm. 2014:17(3):69-75
22. González R, Verdú J. Calidad de vida relacionada con heridas crónicas. Gerokomos. 2010;21(3):131-9. Disponible en: http:// www.gerokomos.com/wp-content/uploads/2015/01/21-3-2010131-helcos.pdf

23. Bastidas M, Pérez F, Torres J, Escobar G, Arango A, Peñaranda F. El diálogo de saberes como posición humana frente al otro: referente ontológico y pedagógico en la educación para la salud. Invest Educ Enferm. [citado 11 de diciembre de 2017]·2009-27(1):104-11.

24. Ferreira N, Souza M, Costa D, Silva A. Integridade cutâneo-mucosa: implicações para a família no cuidado domiciliário ao doente com câncer. Rev. enferm. UERJ 2009:17(2):246-51. Disponible en: http://www.facenf.uerj.br/v17n2/v17n2a19.pdf

25. Rocha M. Pé diabético: fatores comportamentais para a sua prevenção. Tesis de doctorado. Ribeirão Preto: Universidade de São Paulo; 2005.

26. Fuentes A. Intervención Enfermera sobre el nivel de conocimientos del paciente en cuidados postquirúrgicos. Rev. cienc. cuidad. 2017. Disponible en: http://www.imbiomed.com.mx/1/1/articulos.php?method=print\&id_revista=260\&id_seccion=4224\&id ejemplar=10714\&id_articulo=109896

27. Liberato SMD, Araújo $R$ de 0 e, Souza AJG de, Pergola-Marconato AM, Costa IKF, Torres G de V. Adesão ao tratamento de pessoas com úlceras venosas atendidas na atenção primária à saúde. Aquichan [Internet]. 2017 [citado 11 de diciembre de 2017]:17(2):128-39. Disponible en: http://aquichan.unisabana. edu.co/index.php/aquichan/article/view/5296/pdf

28. Miyar LO. Impacto de un programa de promoción de la salud aplicado por enfermería a pacientes diabéticos tipo 2 en la comunidad. Rev Lat Am Enfermagem [Internet]. 2003 Dec [citado 11 de diciembre de 2017];11(6):713-9. Disponible en: http://www.scielo.br/ scielo.php?script=sci_arttext\&pid=S0104-11692003000600003\& $n g=e s \&$ tlng=es

29. Driver VR, Fabbi M, Lavery LA, Gibbons G. The costs of diabetic foot: the economic case for the limb salvage team. J Vasc Surg. 2010:52(3 Suppl):17S-22S. doi: 10.1016/j.jvs.2010.06.003

30. Barboza W. Ejercicio de la disciplina de Enfermería para enfrentar los nuevos retos en salud. Enfermería en Costa Rica. [Internet]. 2012 [citado 11 de diciembre de 2017];33(1). Disponible en: http:// www.binasss.sa.cr/revistas/enfermeria/v33n1/editorial.pdf

31. Giraudo N, Chiarpenello J. Educación para la salud basada en la comunidad (segunda entrega). Evid Act Pract Ambul. 2012;15:21 5. Disponible en: http://www.profam.org.ar/files/632deed00f206e3ae33615c4db104723.pdf 